\title{
Monocular Diplopia: An Optical Correction Modality
}

\author{
Haile W. Alemu ${ }^{a}$ Preetam Kumar ${ }^{b}$ \\ aDepartment of Optometry, College of Medicine and Health Sciences, University of Gondar, \\ Gondar, Ethiopia; 'b.V.Prasad Eye Institute, Kallam Anji Reddy Campus, Hyderabad, India
}

\author{
Keywords \\ Diplopia $\cdot$ Irregular pupil $\cdot$ Contact lens $\cdot$ Trauma $\cdot$ Anisocoria
}

\begin{abstract}
Post-surgical or traumatic corectopia is among the rare causes of monocular diplopia. A 26 -years-old student presented to the Institute with a complaint of monocular double vision in the left eye. He had a penetrating ocular injury in the left eye and subsequently, undergone for multiple ocular surgeries. Following the final intraocular lens implantation, he experienced a monocular double vision in his left eye. Upon contact lens clinic presentation, visual acuities were 20/20 in the right and 20/320 in the left eye (improved to 20/25 with pinhole). Slit-lamp examination on the left eye revealed scarring in the superior nasal quadrant of the cornea, irregular mid-dilated pupil with exposed aphakic and pseudophakic portions. A range of different optical management options were implemented to eliminate monocular diplopia and to correct refractive error. Finally, a combination of prosthetic soft contact lens and spectacle correction was able to remove diplopia and provide binocular single vision.
\end{abstract}

(C) 2021 The Author(s).

Published by S. Karger AG, Basel

\section{Introduction}

Double vision (diplopia) is a symptom resulting from the perception of 2 images of a single object in 1 or both the eyes [1]. It is a symptom with very different etiologies and can occur in 1 or both the eyes. It can be caused by ocular pathology, orbit abnormality, extraocular muscles imbalance, neuromuscular junction abnormality or central nervous system defect [2]. The prevalence after cataract surgery seems to be rare, but it has a distressing symptom and can appear as a major surprise to the patient $[1,2]$.

The management approach can be grossly categorized as surgical or non-surgical and would depend on the cause. Non-surgical interventions such as the correction of vision with spectacles and/or contact lens can produce an excellent visual outcome and an acceptable 
Alemu and Kumar: Diplopia Optical Correction

aesthetic appearance. The optimum contact lens of choice could be scleral lenses, rigid corneal lenses or soft contact lenses [3,4]. This case report describes the management options of monocular diplopia secondary to post-surgical anisocoria and corectopia.

\section{Case Presentation}

A 26-years-old male student presented to the contact lens clinic with a complaint of double vision. He reported a constant double vision in his left eye (LE) following intraocular lens implantation. The double vision disappeared only when the left eye was closed but remain manifested when the right eye (RE) was closed. Presenting visual acuity (VA) was $20 / 20$ on the RE and 20/320 on the LE. He had an open globe ocular injury with metal object type B, zone -I in the left eye. Post-trauma presenting VA was 20/20 on the RE and counting fingers at $1 \mathrm{~m}$ on the LE (Table 1 ).

Following extensive ocular examination, the left eye underwent for corneal tear repair, pars plana vitrectomy, pars plana lensectomy, and intraocular antibiotic injection under local anesthesia. After 2 months, additional pars plana vitrectomy, endolaser as part of vitro retinal surgery, silicone oil injection, and belt buckle for rhegmatogenous retinal detachment surgery was performed in the same eye. Finally, silicone oil removal and scleral fixed intraocular lens (IOL) implantation was performed. Monocular diplopia was manifested since the time of intraocular lens implantation. One month after surgery, spectacles were prescribed at the district to improve vision. But used only for a few days and discontinued because of worsened double vision problem.

An ophthalmologist referred the patient for further nonsurgical management trials. General examination revealed normal facial symmetry, head posture, ocular position, and alignment. Slit-lamp examination revealed no abnormalities in the RE but the LE exhibited paracentral corneal scaring with surface irregularity, a mid-dilated, sluggish reacting irregular pupil, and Glued scleral fixed IOL in place with exposed margin at 10 o'clock position.

Postoperative 19 months, best spectacle-corrected distance VA was 20/20 with plano power in the RE and 20/30 with $-4.50 \mathrm{Ds} /-3.0 \mathrm{Dc} \times 110$ in the LE. However, the patient was unhappy and reported monocular double vision when the LE was open. Binocular vision assessment tests revealed horizontal monocular diplopia worsen during dim illumination in the left eye. As a result, further nonsurgical diplopia management options were initiated.

Table 1. Left eye visual status during the subsequent visits

\begin{tabular}{|c|c|c|c|c|}
\hline Left eye parameter & Presenting VA & PH vision & Dry refraction & $\begin{array}{l}\text { Subjective } \\
\text { acceptance }\end{array}$ \\
\hline First visit & CF@1 m & $\mathrm{NI}$ & Not performed & Not performed \\
\hline $\begin{array}{l}\text { Post PPV+PPL operative } \\
1 \text { months }\end{array}$ & $20 / 800$ & $\mathrm{NI}$ & +12.0 Ds & $\begin{array}{l}\text { With }+12.0 \text { Ds } \\
\text { VA }=20 / 40\end{array}$ \\
\hline Post PPV, Endolaser SOI 1 day & $20 / 600$ & $20 / 200$ & With + 5.0 Ds $2 \mathrm{VA}=0 / 320$ & Not performed \\
\hline $\begin{array}{l}\text { Post- SOR, IOL implantation } \\
\text { operative } 1 \text { day }\end{array}$ & $20 / 400$ & $20 / 80$ & Not performed & Not performed \\
\hline $\begin{array}{l}\text { Post-IOL implantation } \\
18 \text { months }\end{array}$ & $20 / 320$ & $20 / 30$ & $-6.0 /-3.0 \times 110^{\circ}$ & $\begin{array}{l}-4.5 /-3.0 \times 110^{\circ} \\
V A=20 / 30\end{array}$ \\
\hline $\begin{array}{l}\text { Contact lens visit (post- } \\
\text { operative } 19 \text { month) }\end{array}$ & $20 / 320$ & $20 / 25$ & Over CL $-3.0 /-2.5 \times 110^{\circ}$ & $\begin{array}{l}-3.0 /-2.5 \times 110^{\circ} \\
V A=20 / 20\end{array}$ \\
\hline
\end{tabular}




\section{Discussion}

Monocular diplopia patterns could be constant, intermittent, vertical, horizontal or oblique [5]. It can be caused by uncorrected refractive error, corneal scarring, an irregular corneal surface, keratoconus, dry eye syndrome, iris abnormalities, cataract, malposition of the natural or pseudophakic intraocular lens, a badly fitting contact lens, media opacities [1]. In this particular case as shown in Figure 1, monocular diplopia could be caused by uncorrected refractive error, an irregular and scarred corneal surface, anisocoria, superior-nasal corectopia and exposed aphakic portion of an implanted lens.

Determining the root cause of diplopia supports to select the appropriate correction modalities. The pinhole test supports to differentiate monocular diplopia caused by refractive error from other causes [5]. The patient got relief from the diplopia, and vision improved to $20 / 25$. Consequently, the cause of monocular diplopia was confirmed as purely optical as shown in Figure 2.

Modern surgical techniques minimized diplopia following cataract surgery. However, rarely, it may occur in patients who have post-traumatic cataracts [6]. Post-surgical diplopia surprise can be corrected using either surgical or non-surgical approaches. Repeated intraocular surgeries potentially increase the risk of complications [7]. Therefore, optical approaches using spectacles, contact lenses, refractive surgery, and artificial tears are less complicated procedures [1].

The contact lens-based monocular diplopia correction could be corneal rigid contact lenses, combinations of RGP contact lens and prosthetic soft contact lens (piggyback system), prosthetic contact lens, or a combination of prosthetic soft contact lens and spectacles [8-13].

During clinical examination, the presence of corneal scarring, an irregular corneal surface, and irregular astigmatism might cause monocular diplopia. Corneal origin diplopia is best corrected with RGP contact lenses [9-11]. Therefore, classic RGP contact lens (classic laboratory, Bangalore, India, FP 100 material, spherical design) option was discussed with the patient. The rigidity of FP 100 material satisfactorily masking corneal surface irregularities [14]. The initial contact lens parameters such as base curve and diameter were selected based on the fellow eye's measurement. The first trial lens parameters were BC $7.50 \mathrm{~mm}, \mathrm{BVP}-5.00 \mathrm{DS}$, and TD $10.40 \mathrm{~mm}$. Both the static and dynamic fitting assessments suggested a flat fit. Further 2 trials were performed by modifying a lens base curve and diameter. Using the final lens parameter BC $7.00 \mathrm{~mm}$, BVP -8.75 Ds, and TD $9.20 \mathrm{~mm}$, both the dynamic and static lens fittings suggested an acceptable fit. Adequate edge clearance, smooth and acceptable post blink movement, a stable lens in all directions of gaze, minimal

Fig. 1. Shows slit lampmp photography of the left eye mid-dilated, irrgular pupil and corneal scaring at superior-nasal quadrant.

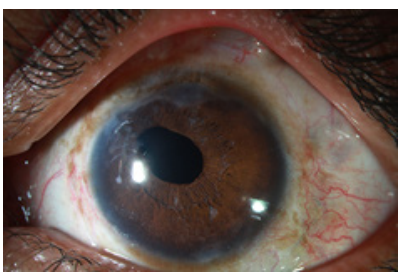

Fig. 2. Shows slit lampmp photography of the left eye irregular pupil with an exposed aphakic portion at superiior-nasal quadrant.

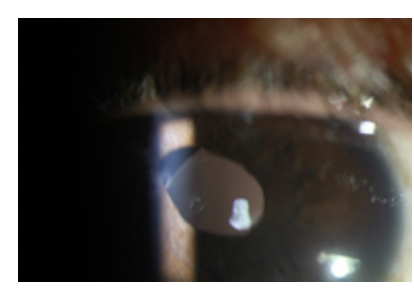


edge lift inferiorly, and optic zone fully covered the pupil. With this RGP contact lens in position VA was improved to 20/20. However, monocular diplopia was still present and annoying the patient. Finally, it was possible to conclude that the monocular diplopia was not corneal in origin.

Due to the mid-dilated peaked pupil at 10 o'clock position, there was an exposed implanted lens margin that permitted light to be refracted through the aphakic and pseudophakic portions simultaneously as shown in Figure 2 . This could potentially cause monocular diplopia.

Another attempt was undertaken to mask pupil irregularity and to make light pass only through the central optical media. To manage this condition, the prosthetic soft contact lens was considered as the best option. Type-C design with hydrogels material considered because of well centered capability, market dominance, a wide range of parameters availability and familiar tinting processes $[15,16]$. Prosthetic soft contact lenses only correct the spherical component of the refractive error. So, any astigmatic component of the refractive error remains to be manifested. Thus, fitting the RGP contact lens over a prosthetic soft contact lens (piggyback system) could overcome the limitations. Prosthetic contact lens aimed to mask irregular pupil and to avoid diplopia and above it RGP contact lens could be applied to mask corneal irregularity. With this system binocular single vision can be achieved. However, this approach has a high risk of corneal complications, the complexity of lens care systems, and limited wearing hours $[13,17]$. Because of all these limitations, the piggyback contact lens system was found to have limited feasibility.

The third option to correct diplopia was by using a combination of prosthetic soft contact lens and spectacles. The combination of a type-C prosthetic soft contact lens and spectacle correction was chosen to avoid diplopia and to correct refractive error respectively. The design of the prosthetic soft contact lens enabled an artificial, small, round pupil which would allow light to be refracted only through the central pseudophakic portion [18] as shown in Figure 3.

Plano type $\mathrm{C}$ medium brown conventional yearly disposable prosthetic soft contact lens (2-polyhydroxy ethyl-metha acrylate, $70 \% \mathrm{H}_{2} \mathrm{O}$ content, manufactured using dot matrix design, Purecon Plc, New Delhi, India) was chosen. A central $3 \mathrm{~mm}$ clear pupil size was chosen to balance illumination, glare, and, moreover, ensured coverage of pupil irregularity. Lens fitting was acceptable, well centered, and completely masked the aphakic portion and the pupil irregularity. Complete limbal coverage and 55\% tightness during lid pushup test was noted. Over refraction was performed and VA was improved to $20 / 20$ using a spectacle power of $-3.00 \mathrm{Ds} /-2.50 \mathrm{Dc} \times 110$. Most importantly it was able to mask the monocular diplopia. The complete coverage of the pupil irregularity allowing incident light only to pass and refracted through the central uniform optical media as shown in Figure 3.

The spherical component of the refractive power was incorporated within the prosthetic soft contact lens. The final contact lens parameters were $7.80 \mathrm{~mm} /-3.00 \mathrm{Ds} / 14.50 \mathrm{~mm}$. Purecon prosthetic, type "C" md brown with $11.80 \mathrm{~mm}$ Iris diameter, $3.0 \mathrm{~mm}$ pupil diameter, and $1.5 \mathrm{~mm}$ clear periphery. Refraction was performed over the contact lens to determine

Fig. 3. Shows slit lampmp photography of the left eye with type-C prosthetic soft contact lens on used to cover pupil irregularity and form artificial round shape.

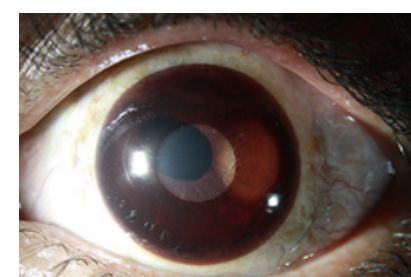


residual cylindrical power and the spectacle power was Plano/-2.25 Dc $\times 110$. Vision improved to 20/20 with no double vision complain. Prosthetic soft contact lens users are strongly advised to wear spectacles in order to correct low degrees of residual refractive error, to maximize the aesthetic appearance and to protect eyes from possible injury [8]. Therefore, spectacles made up of polycarbonate lens material with plastic frames glazed with a plano power for the right eye and corrective power for the left eye prescribed for full-time wear.

In conclusion, identifying the clear cause of monocular diplopia and correct with a proper approach is compulsory. In this case report, a combination of prosthetic soft contact lenses and spectacles sound to eliminate monocular diplopia secondary to post-surgical anisocoria and corectopia.

\section{Statement of Ethics}

The Ethics Committee of the L.V. Prasad Eye Institute, Hyderabad, India, approved this case report and it was conducted in accordance with the tenets of the Declaration of Helsinki. The authors obtained appropriate written informed consent from the patient and permitted to use his ocular images and other clinical information. The patient understands that his medical record number, name and initial will not be published and due efforts will be made to conceal his identity.

\section{Conflict of Interest Statement}

The authors have no conflicts of interest to declare.

\section{Funding Sources}

The authors did not receive any funding.

\section{Author Contributions}

Haile W. Alemu develop the concept and prepare the manuscript. Preetam Kumar initiate the idea, taken pictures and approve the manuscript.

\section{References}

1 Mariana Alves AM, Narciso MR, Luis M, Teresa F. Management of diplopia. Rom J Ophthalmol. 2017;61(3): 166-70.

2 Alves M, Miranda A, Narciso MR, Luis M, Teresa F. Diplopia: a diagnostic challenge with common and rare etiologies. Am J Case Rep. 2015;16:220-3.

3 Chan KY, Cho P, Boost M. Microbial adherence to cosmetic contact lenses. Cont Lens Anterior Eye. 2014;37(4): 267-72.

4 Nilgun Y, Afsun S. Prosthetic contact lenses: adventure or miracle. Eye Contact Lens. 2006;32:102-3.

5 Danchaivijitr C, Kennard C. Diplopia and eye movement disorders. J Neurol Neurosurg Psychiatry. 2004;75(4): iv24-31.

6 Gawęcki M, Grzybowski A. Diplopia as the complication of cataract surgery. J Ophthalmol. 2016;2016:2728712.

7 Maxwell S, Maria A, Jason H, Jeremy D. Scleral-fixated intraocular lenses: past and present. J Vitreoretin Dis. 2017;1:144-52.

8 Mitchell C. Changing lives with prosthetic soft lenses. Contact Lens Spectrum. 2010 Apr 1;25(4):33-7,

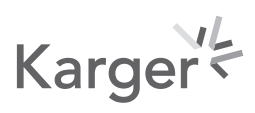


lenses and visual outcome after repaired corneal laceration. Eye Contact Lens. 2015;41:34-9.

10 Rabab M. Rigid gas permeable contact lens as a vision-sparing tool in children after traumatic corneal laceration. J Pediatr Ophthalmol Strabismus. 2018;55(3):178-81.

11 Pradhan ZS, Mittal R, Jacob P. Rigid gas-permeable contact lenses for visual rehabilitation of traumatized eyes in children. Cornea. 2014;33(5):486-9.

12 Sengor T, Kurna SA, Aki S, Ozkurt Y. High Dk piggyback contact lens system for contact lens-intolerant keratoconus patients. Clin Ophthalmol. 2011;5:331-5.

13 Yeung K, Eghbali F, Weissman BA. Clinical experience with piggyback contact lens systems on keratoconic eyes. J Am Optom Assoc. 1995;66(9):539-43.

14 Varsha MR, Srikanth D. Contact lens in keratoconus. Indian J Ophthalmol. 2013;61(8):410-5.

15 David P, Lewis W, Robert T. Tinted contact lenses. IACLE1997.

16 Karen K. Prosthetic soft contact lens and you. Eye Contact Lens. 2005;31(5):215-8.

17 Kok JH, van Mil C. Piggyback lenses in keratoconus. Cornea. 1993;12(1):60-4.

18 Cohen J. Prosthetic contact lens fitting for traumatic mydriasis. Optom J Am Optom Assoc. 2011;82(6):351. 